\title{
Influence of interedge channel scattering on the magneto-transport of 2D-systems
}

\author{
G. Müller, E. Diessel, D. Weiss, K. von Klitzing, K. Ploog \\ Max-Planck-Institut für Festkörperforschung, Heisenbergstrasse I. D-7000 Stuttgart 80, Germany
}

\author{
H. Nickel, W. Schlapp and R. Lösch \\ Forschungsinstitut der Deutschen Bundespost, D-6100 Darmstadt, (jermany
}

Received 31 May 1991: accepted for publication 26 August 1991

\begin{abstract}
We investigate the influcnec of interedge channel scattering (IECS) on adiabatic transport in a two-dimensional electron gas (2DEG) in the quantum Hall regime. To realize adiabatic transport (non-equal distribution of the current among the available edge channels) Schottky gates are used to create areas of different well-defined filling factors. By studying the current dependence one can identify scattering processes and learn how these processes are affected by the device structure. Irradiating the device with photons at the cyclotron resonance frequency in the adiabatic transport regime leads to a drastic change in the magneto-resistance. indicating possible application as a sensitive photoconductor. In addition, a new device structure is discussed allowing direct measurement of the IECS rate.
\end{abstract}

Ten years after the discovery of the quantum Hall effect, there has still been no comprehensive microscopic theory explaining electronic transport in a $2 D E G$ in the quantum Hall regime. However, the new and very clear edge channel (EC) model [1] has opened up a new approach to understand not only the observed resistance quantization in quantum point contacts but also the quantized Hall resistance. Within this model, magneto-transport in the quantum Hall regime is explained in terms of one-dimensional current carrying edge channels formed at the intersection of the Fermi energy with the bent up Landau levels at the edges of the device. Classically these states correspond to skipping orbits moving along the sample boundaries. The contacts are part of the system and are described as carrier reservoirs characterized by an electrochemical potential (ECP). In the quantum Hall regime the number of occupied ECs is given by the filling factor of the 2DEG.
To study the adiabatic transport - which is characterized by a non-equal distribution of the current among the available ECs - it is essential to selectively populate the ECs $[2,3]$. By negatively biasing Schottky gates, we change the transmission and reflection probabilities of the different ECs in a controlled manner. Fig. 1 shows the two device geometries used to study the influence of the applied current on the IECS for the bulk filling factor $b=4[4,5]$. In fig. 1a, the gate with the filling factor $g_{1}=2$ works as injector gate. selectively populating the outer spin degenerate $\mathrm{EC}$, whereas the gate with filling factor $g_{\mathrm{D}}=2$ selectively detects the incoming outer EC (detector gate) $[3,6]$. In fig. 1 the dispersion relation as function of the guiding center coordinate for the intersection $A-B$ is also shown. At $A$ in fig. la, the two spin degenerate ECs are populated up to the ECP of contact 4 , whereas at B assuming complete adiabatic transport, the ECP of the inner $\mathrm{EC}$ is the same as at $\mathrm{A}$. 
To investigate whether there is any asymmetry between the two current directions, we apply the current between the contacts 1 and 3 . By adding a small AC-current of $30 \mathrm{nA}$ to this DC-current, we measure the differential four point Hall resistance $R_{13,24}^{\prime}$ between the probes 2 and 4 . For complete adiabatic transport, one can calculate [3] that the four point Hall resistance $R_{13,24}$ for the sketched situation equals $h / 2 \mathrm{e}^{2}$ whereas for equilibrated transport (cach available EC carrics the same amount of current) $R_{13,24}=h / 4 \mathrm{e}^{2}$. Because with a voltmeter one only measures ECP differences one does not know the ECPs relative to the Landau bands. In the following we assume a symmetric partition of the ECP difference for the outer EC between the two edges compared to $I=0$. Increasing the current in the direction, for which the ECP of the outer EC is smaller than that of the inner $\mathrm{EC}(I<0)$ results in a resistance increase corresponding to increased adiabatic transport. For this experiment two opposing effects have to be considered; the build up of net positive charge [4] in the outer EC at $B$ and the increase of the ECP of the inner EC. The first effect reduces the IECS rate, since the effective positive charge close to the edge bends down the energy level of the outer EC. Therefore the spatial separation between the ECs becomes larger and IECS is more unlikely as seen in the experi- ment. The second effect, depending on the slope of the edge potential increases the IECS rate. At a certain current threshold, the emission of acoustic phonons becomes possible [4] and this new scattering channel increases the IECS rate. Taking the maximum of the differential resistance as the onset of phonon emission, we estimate the spatial separation of the ECs to be 780 $\AA$ (the magnetic length $l_{\mathrm{c}}=190 \AA$ ). We attribute the observed very poor adiabatic transport to strong IECS centers in the path between the injector and the detector gate $(45 \mu \mathrm{m})$. For the other current direction the spatial separation at the ECP of the outer EC is reduced to a greater extent and therefore transport becomes equilibrated.

Fig. 1b shows a symmetric geometry used to investigate adiabatic transport. For the case of $b=4$ and $g=2$ under both Schottky gates, the two ECs are sketched. In between the two gates the inner EC forms a closed loop. As long as there is no IECS, there is no coupling between the inner and outer EC. The whole arrangement works as a single, large gate. If there is IECS, the ECs "see" the bulk filling factor in between the gates and therefore the current is redistributed among the different ECs. The longitudinal four point resistance $R_{14,23}$ is now the series resistance of two individual gates, giving rise to a change by
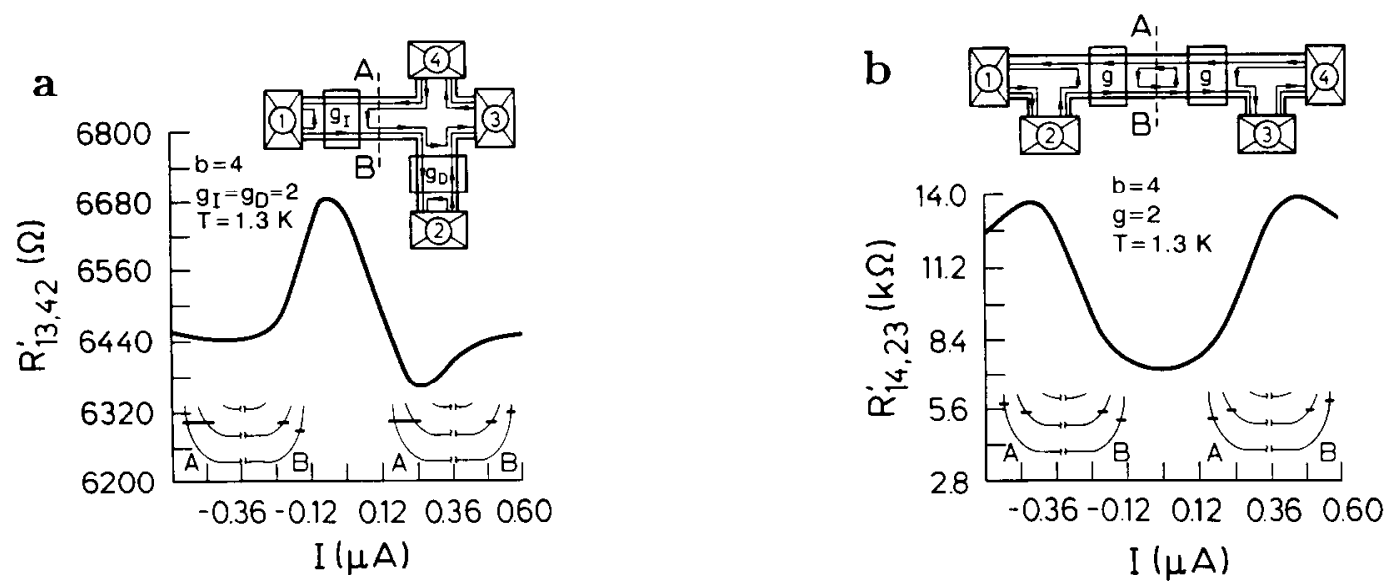

Fig. 1. Differential four point resistance as function of the applied current for the two device geometries (with the ECs sketched). The sample investigated in (a) has a carrier density of $N_{\mathrm{s}}=1.7 \times 10^{11} \mathrm{~cm}^{-2}$ and a mobility $\mu=640000 \mathrm{~cm}^{2} / \mathrm{V} \cdot \mathrm{s}$ at liquid helium temperature, whereas for (b) $N_{\mathrm{s}}=2.0 \times 10^{11} \mathrm{~cm}^{-2}$ and $\mu=1.2 \times 10^{6} \mathrm{~cm}^{2} / \mathrm{V} \cdot \mathrm{s}$. The inset shows schematically the ECP of the different ECs for the two current directions at the intersection $\mathrm{A}-\mathrm{B}$. 
a factor of two compared with complete adiabatic transport $\left(R_{14,23}\right.$ (adiabatic) $=h / 4 \mathrm{e}^{2}, R_{14,23}$ (equilibrated) $\left.=h / 2 \mathrm{e}^{2}\right)$ [3]. These resistance values are exactly opposite to the results for $R_{13,24}$ in fig. 1a. Fig. $1 \mathrm{~b}$ shows the dependence of the differential four point resistance $R_{14,23}^{\prime}$ on the applied current. Increasing the current results in a crossover from adiabatic to equilibrated transport. There is no stronger adiabatic transport for larger currents $(|I|<0.6 \mu \mathrm{A})$ as for the other geometry. In contrast to the cross geometry, scattering at both edges is necessary here to equilibrate the transport. As a consequence, the smaller scattering rate occurring at $\mathrm{B}$ for $I<0$ determines the resistance.

In this device geometry it is easier to observe adiabatic transport than in the cross geometry. Assuming a weak link between the ECs on one sample side results in an equal ECP for the ECs on this side but nevertheless the transport in that structure can be quite adiabatic as long as the IECS rate on the opposite side is sufficiently small. But for such a case one would observe a pronounced asymmetry in the current due to the different microscopic nature of the edge region.

To investigate not only phonon and impurity scattering but also photon induced IECS processes we have irradiated a structure with the geometry of fig. $1 \mathrm{~b}$ (gate finger spacing $\approx 50 \mu \mathrm{m}$ ) with photons from a far infrared laser. Fig. 2 shows the photoconductivity signal $\Delta R_{14,23}$ at a laser intensity of $I=10^{-6} \mu \mathrm{W} \mathrm{cm}{ }^{-2}$ as function of the magnetic field for an AC-current of 100 $\mathrm{nA}$. The laser wavelength of $\lambda=232 \mu \mathrm{m}$ leads to cyclotron resonance absorption at about $B \approx 3.2$ T. Trace (a) demonstrates the striking performance of our photoconductor in the adiabatic transport regime. If the ECs are reflected at both barriers (e.g., a gate voltage of $-220 \mathrm{mV}$ leads at the resonance magnetic field to $b \approx 3.5$ and $g=1$ ) a strong photosignal is observed. For the case of no gate voltage applied (trace (b)) there is only a small dependence on the magnetic field. If one applies $-220 \mathrm{mV}$ to only one gate (trace (c)), no change of $\Delta R_{14,23}$ due to IECS is expected giving the weak observed signal. We conclude that in the two-gate case, the photons raise the IECS rate which is reflected by the huge increase in the

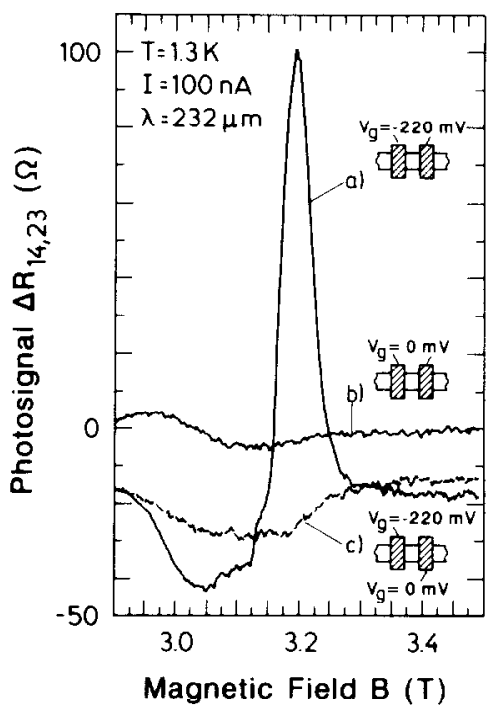

Fig. 2. Photoconductivity signal due to cyclotron resonance photons as function of the magnetic field for a sample with $N=2.7 \times 10^{11} \mathrm{~cm}^{2}$ and $\mu=560000 \mathrm{~cm}^{2} / \mathrm{V} \cdot \mathrm{s}$.

resistance. Further support for the concept of strongly enhanced photoconductivity in the adiabatic transport regime is demonstrated by the current and temperature dependence of the peak height. We observe a reduced photosignal by raising the current or the temperature which can be attributed to enhanced IECS (discussion above and ref. [5]).

In the last section we would like to present a new geometry which allows a direct correlation between the measured current and the IECS

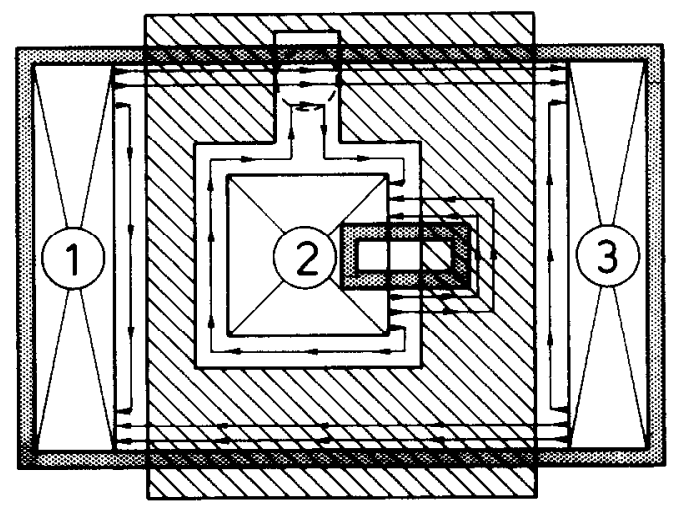

Fig. 3. Schematic layout of a new device structure with at pseudo corbino contact to study IECS. 
rate. The geometry (fig. 3) consists of two conventional ohmic contacts $(1,3)$ and one pseudo corbino contact (2) surrounded by a gate. A special feature is the etched hole which connects the interior contact with the gate. Fig. 3 also shows schematically the ECs for $b=3$ and $g=2$. Contacts 1 and 3 both emit 3 ECs, the outer ones are transmitted and only the inner is reflected back by the gate. The inner contact 2 also injects 3 ECs, two of which run around the etched hole back to contact 2 , whereas the inner one is reflected at the gate and returns along the ungated region to contact 2 . This is the first geometry where an ohmic contact is electrically controlled and hence only accessible to an inner $\mathrm{EC}$. If one assumes complete adiabatic transport for the situation sketched in fig. 3 no current flow is possible for an applied voltage between the contacts 1 and 2. The inner contact becomes connected to the "outer" world only in the presence of IECS. IECS forces the inner contact to make a transition from a corbino contact to a contact with electrical access. A calculation of the two-terminal resistance between the inner and one of the outer contacts gives an infinite result for complete adiabatic transport and $R=\left(h / \mathrm{e}^{2}\right)\{b /[g \times$ $(b-g)]\}$ for equilibrated transport. The twoterminal resistance is directly proportional to the IECS rate as long as the measured resistance is much larger than the equilibrated value. On its main path IECS is not possible, because the inner
$\mathrm{EC}$ is well separated from the others. IECS is only possible in the region defined by the dashed circle. Preliminary results indicate two point resistances as high as $200 \mathrm{k} \Omega$ at $T=1.3 \mathrm{~K}$.

In summary, we have discussed the current dependence of adiabatic transport in different geometries elucidating IECS. Resonant photon induced IECS has been investigated using photoconductivity experiments, providing a new method of equilibrating the transport. Finally we have proposed a device geometry to directly measure the IECS rate.

\section{References}

[1] M. Büttiker, Phys. Rev. Lett. 57 (1986) 1761; Phys. Rev. B 38 (1988) 9375.

[2] B.J. van Wees, E.M.M. Willems, K.J.P.M. Harmans, C.W.J. Beenakker, H. van Houten, J.G. Williamson, C.T. Foxon and J.J. Harris, Phys. Rev. Lett. 62 (1989) 1181;

S. Komiyama and H. Hirai, Phys. Rev. B 40 (1989) 7767.

[3] G. Müller, D. Weiss, S. Koch, K. von Klitzing, H. Nickel, W. Schlapp and R. Lösch, Phys. Rev. B 42 (1990) 7633.

[4] S. Komiyama, H. Hirai, M. Ohsawa, H. Matsuda, S. Sasa and T. Fujii, Proc. 20th Int. Conf. Phys. Semiconductors, Eds. E.M. Anastassakis and J.D. Joannopoulos (World Scientific, Singapore, 1990) p. 1150.

[5] G. Müller, D. Weiss, S. Koch, K. von Klitzing, H. Nickel, W. Schlapp and R. Lösch, Proc. 20th Int. Conf. Phys. Semiconductors, Eds. E.M. Anastassakis and J.D. Joannopoulos (World Scientific, Singapore, 1990) p. 829.

[6] B.W. Alphenaar, P.L. McEuen, R.G. Wheeler and R.N. Sacks, Phys. Rev. Lett. 64 (1990) 677. 